\title{
On the User Selection for MIMO Broadcast Channels
}

\author{
Alireza Bayesteh and Amir K. Khandani \\ Department of Electrical Engineering \\ University of Waterloo, Waterloo, ON, N2L 3G1 \\ Email: \{alireza,khandani\}@ cst.uwaterloo.ca
}

\begin{abstract}
In this paper, we consider a downlink communication system, in which a base station (BS) equipped with $M$ antennas communicates with $N$ users each equipped with $K$ receive antennas. We propose an efficient suboptimum algorithm for selecting a set of users in order to maximize the sum-rate throughput of the system. For the asymptotic case of $N \rightarrow \infty$, it is shown that by using a very simple precoding scheme of zeroforcing beam-forming, the optimum sum-rate which behaves like $M \log \log N$ can be achieved. The complexity of our algorithm is investigated in terms of the required amount of feedback from the users to the base station, as well as the number of searches required for selecting the users. It is shown that the proposed method is capable of achieving a large portion of the sum-rate capacity, with a very low complexity ${ }^{2}$.
\end{abstract}

\section{INTRODUCTION}

Many recent applications demand for transmission of data at high rates. Multiple-input multiple-output (MIMO) systems have proved their ability to achieve high bit rates on a scattering wireless network [2].

In a MIMO Broadcast Channel (MIMO-BC), the base station equipped with multiple antennas communicates with several multiple-antenna users. Recently, there has been lots of interest in characterizing the capacity region of this channel [3], [4], [5], [6]. In [3]-[5], it has been shown that the sumrate capacity of MIMO-BC can be achieved by applying Dirty Paper Coding (DPC) [7] at the transmitter. Practical schemes for implementation of DPC are proposed in [8], [9], [10]. However, achieving the theoretical limits promised by DPC faces many challenges.

In a network with a large number of users, the base station can increase the throughput by selecting the best set of users to communicate with. This results in the so-called "multiuser diversity" gain [11], [12]. However, achieving the optimum multiuser diversity gain requires an exhaustive search over all possible combination of the users, which is not practical for large-scale networks. To overcome this problem, references [13] and [14] propose sub-optimum methods for user selection. These methods exploit the multiuser diversity gain, but are based on assuming DPC at the base station.

To avoid the complexity of the DPC, the simple precoding scheme of "zero-forcing beam-forming", which is sometimes

\footnotetext{
${ }^{1}$ This work is financially supported by Communications and Information Technology Ontario (CITO), Nortel Networks, and Natural Sciences and Engineering Research Council of Canada (NSERC).

${ }^{2}$ More details about this work can be found in [1]
}

called "channel inversion", is considered by some authors [15], [16], [17], [18]. Using zero-forcing beam-forming, the downlink channel with $M$ transmit antennas is decomposed into $N \leq M$ interference-free subchannels, serving $N$ users. To achieve a good performance by using zero-forcing beamforming, the selected sub-channels must have high gains and be nearly orthogonal to each other. As the number of users increases, it becomes easier to meet such requirements. However, the exhaustive search for selecting the best set of users is very complex. In [19], the authors propose a suboptimum algorithm for selecting such a set of users in a downlink environment with large number of single-antenna users. It has been shown that using this algorithm, the optimum sum-rate throughput of the system can be asymptotically achieved as $N \rightarrow \infty$. However, in their approach, the base station must have perfect channel state information (CSI) for all users.

To avoid the huge amount of feedback required by providing perfect CSI to the base station, reference [20] proposes a downlink transmission scheme based on random beamforming relying on partial CSI at the transmitter. Reference [20] shows that when the number of users tends to infinity, the optimum sum-rate throughput can be achieved. However, for practical number of users, it does not perform well [19].

In this paper, we consider a MIMO-BC with large number of users and propose an efficient sub-optimum algorithm that assigns the coordinates of transmission space to different users in order to achieve the best performance in terms of the sumrate throughput. Zero-forcing beam-forming is used at the base station as the precoding scheme. The algorithm starts by setting a threshold value. By applying Singular Value Decomposition (SVD) to all users' channel matrices, only the eigenvectors whose corresponding singular values are above the set threshold are considered. Then, among these candidate eigenvectors, the algorithm chooses a set of size $M$ which are nearly orthogonal to each other. Our analysis shows that this scheme achieves the optimum sum-rate capacity for $N \rightarrow \infty$, which scales as $M \log \log N$. The advantage of our algorithm over the algorithm proposed in [19] is that the coordinates are selected among the eigenvectors with singular values above a certain threshold, and the rest do not require to feedback their information to the base station. Therefore, the complexity of search and the amount of feedback is decreased significantly.

This paper is organized as follows. In section II, we introduce the system model, and the proposed algorithm is 
described in section III. Sections IV and V are devoted to analyzing the performance, in terms of sum-rate throughput, and the complexity of our proposed algorithm, respectively. Finally, section VI concludes the paper.

Throughout this paper, the norm of the vectors are denoted by $\|$.$\| , the Hermitian operation is denoted by (.)^{*}$, and the determinant and the trace operations are denoted by $\operatorname{det}($.$) and$ $\operatorname{Tr}($.$) , respectively. \mathbb{E}\{$.$\} represents the expectation, notation$ "log" is used for the natural logarithm, and the rates are expressed in nats.

\section{System Model}

In this work, a MIMO-BC in which a base station equipped with $M$ antennas communicates with $N$ users, each equipped with $K$ antennas, is considered. We assume a homogenous network, where the channel between each user and the base station is modeled as a zero-mean circularly symmetric Gaussian matrix (Rayleigh fading). The received vector by user $k$ can be written as

$$
\boldsymbol{y}_{k}=\boldsymbol{H}_{k} \boldsymbol{x}+\boldsymbol{n}_{k},
$$

where $\boldsymbol{x} \in \mathbb{C}^{M \times 1}$ is the transmitted signal, $\boldsymbol{H}_{k} \in \mathbb{C}^{K \times M}$ is the channel matrix from the transmitter to the $k$ th user (assumed to be known at the receiver side), and $\boldsymbol{n}_{k} \in \mathbb{C}^{K \times 1} \sim$ $\mathcal{C N}\left(\mathbf{0}, \boldsymbol{I}_{K}\right)$ is the noise vector for the $k$ th user. We assume that the transmitter has an average power constraint $P$, i.e. $\mathbb{E}\left\{\operatorname{Tr}\left(\boldsymbol{x} \boldsymbol{x}^{*}\right)\right\} \leq P$. We consider a block fading model in which each $\boldsymbol{H}_{k}$ is constant for the duration of a frame. The frame itself is assumed to be long enough to allow communication at rates close to the capacity.

The optimum achievable sum-rate capacity in MIMO-BC is equal to [3]

$\mathcal{R}_{\mathrm{Opt}}=\mathbb{E}\left\{\max _{\substack{\boldsymbol{Q}_{n} \\ \sum \operatorname{Tr}\left(\boldsymbol{Q}_{\mathrm{n}}\right)=\mathrm{P}}} \log \operatorname{det}\left(\boldsymbol{I}_{M}+\sum_{n=1}^{N} \boldsymbol{H}_{n}^{*} \boldsymbol{Q}_{n} \boldsymbol{H}_{n}\right)\right\}$,

where $\boldsymbol{Q}_{n}$ is the transmit covariance matrix of the $n$th user, and the expectation is taken over the channel matrices $\boldsymbol{H}_{1}, \cdots, \boldsymbol{H}_{N}$.

\section{Proposed Algorithm}

As mentioned earlier, to maximize the sum-rate using zero-forcing beam-forming, the selected eigenvectors must be nearly orthogonal to each other, and their corresponding singular values be sufficiently large. Basically, the measure of orthogonality between two $M \times 1$ vectors $\boldsymbol{v}$ and $\boldsymbol{\psi}$ is defined as,

$$
z(\boldsymbol{v}, \boldsymbol{\psi})=\frac{\left|\boldsymbol{v}^{*} \boldsymbol{\psi}\right|^{2}}{\|\boldsymbol{v}\|^{2}\|\boldsymbol{\psi}\|^{2}}
$$

It is evident that the smaller is $z(\boldsymbol{v}, \boldsymbol{\psi})$, the more orthogonal will be $v$ and $\psi$.

Using Singular Value Decomposition (SVD), $\boldsymbol{H}_{k}$ can be written as

$$
\boldsymbol{H}_{k}=\boldsymbol{U}_{k} \boldsymbol{\Lambda}_{k} \boldsymbol{V}_{k}^{*}
$$

where $\boldsymbol{\Lambda}_{k}$ is an $K \times M$ diagonal matrix containing the singular values of $\boldsymbol{H}_{k}, \boldsymbol{U}_{k}$ and $\boldsymbol{V}_{k}$ are $K \times K$ and $M \times M$ unitary matrices, respectively. Multiplying both sides of (1) by $\boldsymbol{U}_{k, j}^{*}$, where $\boldsymbol{U}_{k, j}$ is the $j$ th column of $\boldsymbol{U}_{k}$, it is easy to show that

$$
r_{k, j}=\boldsymbol{g}_{k, j} \boldsymbol{x}+w_{k, j} .
$$

In the above equation, $r_{k, j}=\boldsymbol{U}_{k, j}^{*} \boldsymbol{y}_{k}, \boldsymbol{g}_{k, j}=\sqrt{\lambda_{j}(k)} \boldsymbol{V}_{k, j}^{*}$, where $\boldsymbol{V}_{k, j}$ is the $j$ th column of $\boldsymbol{V}_{k}$ and $\sqrt{\lambda_{j}(k)}$ is the $j$ th singular value of $\boldsymbol{H}_{k}$ corresponding to $\boldsymbol{V}_{k, j}$, and $w_{k, j} \sim \mathcal{C N}(0,1)$ is an AWGN. This equation suggests that for selecting the dimensions with high gains, the norm of the equivalent channel introduced by (5), $\boldsymbol{g}_{k, j}$, which is equal to $\sqrt{\lambda_{j}(k)}$, can be compared with a threshold. This threshold is set by the base station at the beginning of the transmission. Using such a threshold, also reduces the amount of feedback and the size of search space for selecting the coordinates. To satisfy the orthogonality criterion, the base station can perform an exhaustive search for finding the "most orthogonal set"3 among the pre-selected eigenvectors. Due to the huge complexity of exhaustive search, the coordinates can be chosen one by one. In other words, in each step the eigenvector which is the most orthogonal to the previously selected coordinates, is selected. The first coordinate is chosen as the eigenvector with the maximum corresponding singular value. The steps of the algorithm can be explained in the following:

\section{Proposed Algorithm (Algorithm 1):}

1. Using SVD, each user computes the eigenvectors and singular values of its channel matrix and sends back the singular values which are larger than a predetermined threshold $t$, along with their corresponding "right" eigenvectors, to the base station. These eigenvectors form the following set:

$$
\mathcal{S}_{0}=\left\{(k, j) \mid \quad \lambda_{j}(k)>t\right\} .
$$

2. Base station selects the index corresponding to the maximum value in $\mathcal{S}_{0}$. Let us define this index as $\left(s_{1}, d_{1}\right)$, i.e., the $d_{1}$ th dimension of the $s_{1}$ th user.

3. Define

$$
\begin{aligned}
\mathcal{S}_{1} & =\mathcal{S}_{0}-\left\{\left(s_{1}, d_{1}\right)\right\}, \\
\gamma_{k, j}(1) & =z\left(\boldsymbol{V}_{s_{1}, d_{1}}, \boldsymbol{V}_{k, j}\right) \quad \forall(k, j) \in \mathcal{S}_{1},
\end{aligned}
$$

where $z(.,$.$) is defined in (3). Note that as \left\|\boldsymbol{V}_{k, j}\right\|=$ $\left\|\boldsymbol{V}_{s_{i}, d_{i}}\right\|=1, z\left(\boldsymbol{V}_{s_{i}, d_{i}}, \boldsymbol{V}_{k, j}\right)=\left|\boldsymbol{V}_{s_{m}, d_{m}}^{*} \boldsymbol{V}_{k, j}\right|^{2}$.

4. For $2 \leq m \leq M$, repeat the followings:

$$
\begin{aligned}
\left(s_{m}, d_{m}\right) & =\arg \min _{(k, j) \in \mathcal{S}_{m-1}} \gamma_{k, j}(m-1) \\
\mathcal{S}_{m} & =\mathcal{S}_{m-1}-\left\{\left(s_{m}, d_{m}\right)\right\} \\
\gamma_{k, j}(m)= & z\left(\boldsymbol{V}_{s_{m}, d_{m}}, \boldsymbol{V}_{k, j}\right)+\gamma_{k, j}(m-1), \\
& \forall(k, j) \in \mathcal{S}_{m}
\end{aligned}
$$

${ }^{3}$ In general, the orthogonality of a set $\left\{\boldsymbol{h}_{i}\right\}_{i=1}^{M}$ can be measured by the orthogonality defect, defined as $\frac{\prod_{i=1}^{M}\left\|\boldsymbol{h}_{i}\right\|^{2}}{\operatorname{det}\left[\boldsymbol{H} \boldsymbol{H}^{*}\right]}$, where $\boldsymbol{H}=\left[\boldsymbol{h}_{1}^{T}|\cdots| \boldsymbol{h}_{M}^{T}\right]^{T}$. 
In the above, $\gamma_{k, j}(m-1)=\sum_{i=1}^{m-1} z\left(\boldsymbol{V}_{s_{i}, d_{i}}, \boldsymbol{V}_{k, j}\right)$ is used as the measure of orthogonality between a candidate eigenvector $\boldsymbol{V}_{k, j}$ and the set of selected eigenvectors, $\left\{\left(s_{i}, d_{i}\right)\right\}_{i=1}^{m-1}$, up to the $m$ th step. Since these dimensions are nearly orthogonal to each other by the algorithm, with a good approximation, $\gamma_{k, j}(m-1)$ can be interpreted as the square magnitude of the normalized projection of $\boldsymbol{V}_{k, j}$ over the sub-space spanned by $\left\{\boldsymbol{V}_{s_{i}, d_{i}}\right\}_{i=1}^{m-1}$. It is obvious that the smaller is this projection, the more orthogonal will be $\boldsymbol{V}_{k, j}$ to this sub-space. The recursive structure of $\gamma_{k, j}(m)$, facilitates its computation at each step of the algorithm.

After selecting the users we construct the "selected coordinate matrix" as

$$
\boldsymbol{H}(\mathcal{S})=\left[\boldsymbol{g}_{s_{1}, d_{1}}^{T}\left|\boldsymbol{g}_{s_{2}, d_{2}}^{T}\right| \cdots \mid \boldsymbol{g}_{s_{M}, d_{M}}^{T}\right]^{T} .
$$

Using zero-forcing beam-forming, the transmitted vector $\boldsymbol{x}$ can be written as

$$
\boldsymbol{x}=\boldsymbol{H}(\mathcal{S})^{-1} \boldsymbol{u},
$$

where $\boldsymbol{u}=\left[u_{s_{1}, d_{1}}, \cdots, u_{s_{M}, d_{M}}\right]^{T}$ is the intended vector to be sent over the selected coordinates. Using (5) and (10), the received signal over the $m$ th coordinate is equal to

$$
\begin{aligned}
r_{s_{m}, d_{m}} & =\boldsymbol{U}_{s_{m}, d_{m}}^{*} \boldsymbol{y}_{k} \\
& =u_{s_{m}, d_{m}}+w_{s_{m}, d_{m}} .
\end{aligned}
$$

It can be seen that by applying zero-forcing beam-forming the downlink channel is decomposed to $M$ interference-free sub-channels.

\section{Performance Analysis}

In this section, we examine the performance of our proposed algorithm in terms of the sum-rate throughput. First, we consider the asymptotic case of $N \rightarrow \infty$.

\section{A. Asymptotic Analysis}

The sum-rate capacity of MIMO-BC has been shown to scale like $M \log \log N$, as $N$ tends to infinity [20]. This implies that the singular values ${ }^{4}$ corresponding to the selected eigenvectors should behave like $\log N$. Here, we set the threshold $t=\log N+(K-2) \log \log N .^{5}$

The following lemma implies that with this threshold, at most one dimension is likely to be selected from a given user.

Lemma 1- Assuming $K>1$, define $\Omega_{J}$ as the probability of existing at least one user with $J$ dimensions $(J \leq K)$ being selected using the proposed algorithm. We have

$$
\Omega_{J} \sim O\left(\frac{[\log N]^{M K-1}}{N^{J-1}}\right) .
$$

As a result, $\lim _{N \rightarrow \infty} \Omega_{J}=0$, for $J>1$. This implies that as $N \rightarrow \infty$, with probability one, at most one eigenvector for any user is likely to be selected by this algorithm. This eigenvector

\footnotetext{
${ }^{4}$ We mean the singular values of $\boldsymbol{H} \boldsymbol{H}^{*}$.

${ }^{5}$ The threshold is set to this value in order to reduce the amount of feedback, while guaranteeing the $\frac{1}{\log N}$-orthogonality among the users to be selected in the next step.
}

must correspond to the maximum singular value of that user. The following lemma gives the asymptotic number of users whose maximum singular values are above $t$ :

Lemma 2- Let $L$ be the number of users being selected in the first step of algorithm 1. Then, as $N \rightarrow \infty$, with probability one

$$
L=\frac{[\log N]^{M}}{\Gamma(M) \Gamma(K)}\left(1+O\left(\frac{\log \log N}{\log N}\right)\right) .
$$

After pre-selecting the dimensions with high gains, the algorithm searches for a subset of $M$ nearly orthogonal dimensions, among those candidates. To do this, the algorithm uses the orthogonality measure defined in (3). As shown in Lemma 1, in the asymptotic case of $N \rightarrow \infty$ at most one eigenvector from each user is likely to be selected. This eigenvector must correspond to the maximum singular value of that user's channel matrix, and is denoted by $\boldsymbol{V}_{i, \max }$. Hence, for the sake of simplicity of notation, we define the measure of orthogonality between the users $i$ and $j$, denoted by $z(i, j)$, as the orthogonality measure between $\boldsymbol{V}_{i, \max }$ and $\boldsymbol{V}_{j, \max }$. In other words,

$$
z(i, j)=\left|\boldsymbol{V}_{i, \max }^{*} \boldsymbol{V}_{j, \max }\right|^{2} .
$$

From [1], Appendix A, the probability density function of $z(i, j)$ can be computed as,

$$
p_{z(i, j)}(z)=(M-1)(1-z)^{M-2} .
$$

Definition- $A$ set $\mathcal{S}=\left\{\boldsymbol{\psi}_{i}\right\}_{i=1}^{M}$, in which $\boldsymbol{\psi}_{i} \in \mathbb{C}^{1 \times M}$, is called $\epsilon$-orthogonal, if we have $z\left(\boldsymbol{\psi}_{i}, \boldsymbol{\psi}_{j}\right)<\epsilon$, for every $\boldsymbol{\psi}_{i} \neq \boldsymbol{\psi}_{j} \in \mathcal{S}$.

Theorem1- As $N \rightarrow \infty$, the selected users by the proposed algorithm, with probability one, construct an $\frac{1}{\log N}$-orthogonal set.

Proof- Refer to [1].

Theorem 1 implies that as the number of users increases, the selected coordinates become more and more orthogonal to each other, and in the limit of $N \rightarrow \infty$, they construct an orthogonal basis for $\mathbb{C}^{M}$. This result is not surprising, since the number of users out of which this set is selected tends to infinity.

The following theorem which is proved in [1], shows the optimality of our proposed method in the asymptotic case of $N \rightarrow \infty$ :

Theorem 2-Consider a MIMO-BC in which a base station with $M$ transmit antennas, and total power constraint $P$ communicates with $N$ users, each equipped with $K$ antennas. Then as $N \rightarrow \infty$, using the proposed algorithm and applying zero-forcing beam-forming at the base station, the optimum sum-rate capacity can be achieved, i.e.,

$$
\lim _{N \rightarrow \infty} \mathcal{R}_{\text {Prop }}-\mathcal{R}_{\text {Opt }}=0 .
$$

Theorem 2 implies that using the proposed algorithm for user selection, and applying zero-forcing beam-forming at the base station, the same performance as when the optimum user selection algorithm, and optimum precoding scheme is utilized, can asymptotically be achieved. 


\section{B. Simulation Results}

In this section, we provide some numerical results to evaluate the performance of our scheme.

Figures 1-4 represent the graphs of sum-rate capacity for our proposed scheme (with using zero-forcing beam-forming), as well as the optimal scheme (using DPC) and TDMA (Time Division Multiple Access). The Signal to Noise Ratio (SNR), which is equal to the transmitted power $P$, is fixed at $10 \mathrm{~dB}$ in these figures. Also, in all simulations, the optimum value for the threshold is used. For the cases that the base station and the users have 2 antennas (figures 1 and 2), it can be observed that the performance of our scheme is very close to the optimum performance. In the case that the base station has 4 antennas (figures 3 and 4), there is a gap between the same-rate achieved by our method and the optimum sum-rate, but the performance is still acceptable (especially for the case $M=K=4)$. Moreover, it is observed that TDMA method has a weak performance in all cases (refer to [1] for more details about TDMA).

Figure 5 represents the plots of sum-rate capacity versus SNR, for $M=2, K=1$ and $M=4, K=1$. The number of users is fixed to 100 in this case. It can be observed that the proposed scheme has a good performance for all SNRs.

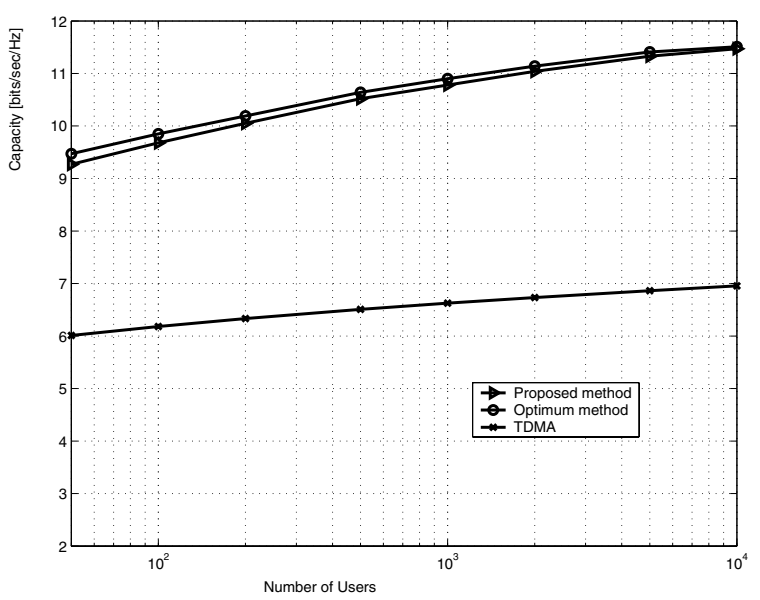

Fig. 1. Sum-rate capacity, $M=2, K=1$

\section{COMPleXity ANALYSiS}

The use of zero-forcing beam-forming at the base station, has significantly decreased the complexity of precoding in the proposed algorithm. In this section, we investigate the complexity of our algorithm in terms of the amount of feedback required from the users to the base station, and the number of searches required for selecting the best set of users.

\section{A. Amount of Feedback Required}

As can be observed in the proposed algorithm, only the eigenvectors that belong to $\mathcal{S}_{0}$, defined in (6), must be sent back to the base station, along with their corresponding singular values. For the asymptotic case of $N \rightarrow \infty$, from Lemma 2 , we conclude that the cardinality of $\mathcal{S}_{0}$ scales as $\frac{[\log N]^{M}}{\Gamma(M) \Gamma(K)}$. Assuming that for each eigenvector and its singular

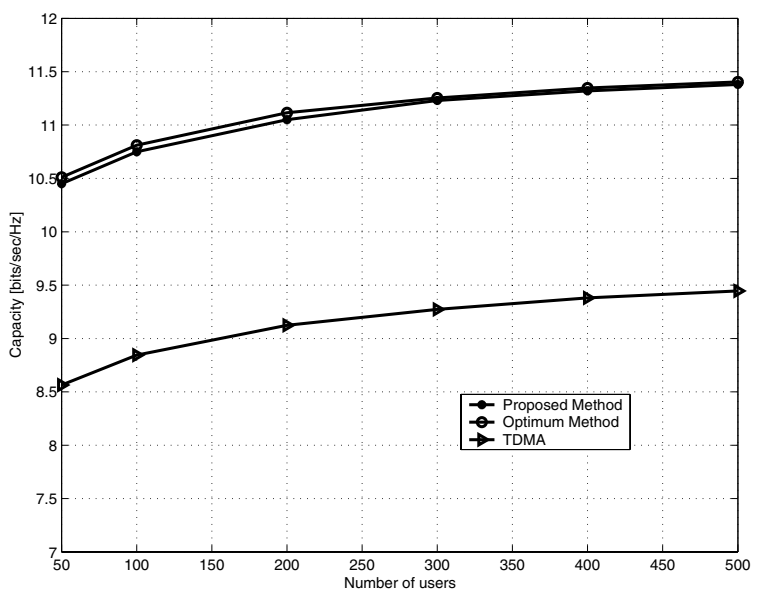

Fig. 2. Sum-rate capacity, $M=2, K=2$

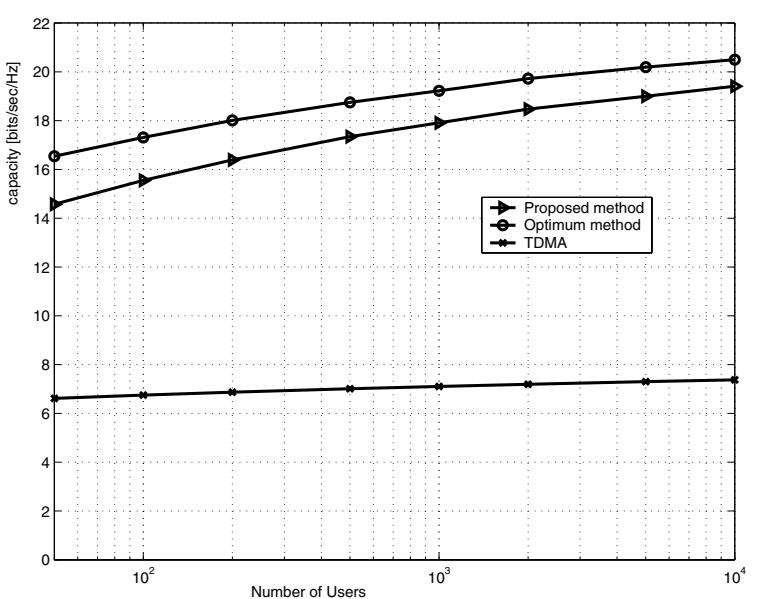

Fig. 3. Sum-rate capacity, $M=4, K=1$

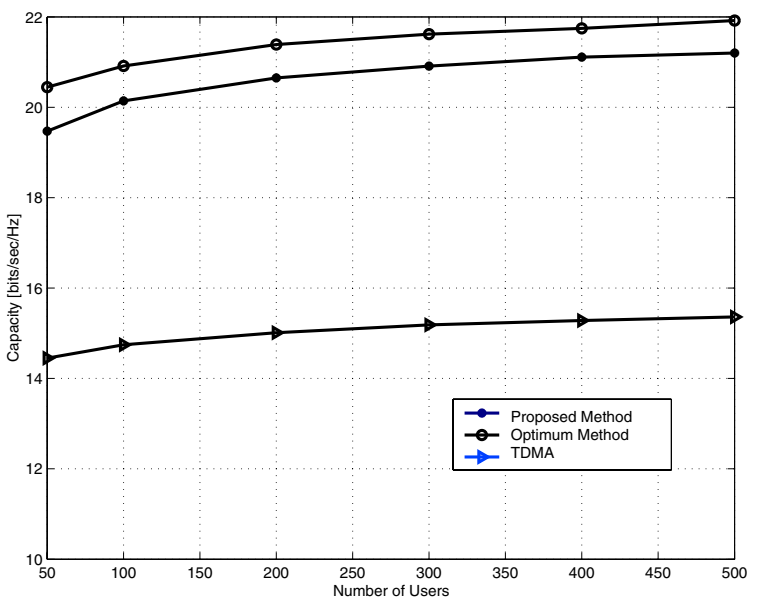

Fig. 4. Sum-rate capacity, $M=4, K=4$ 


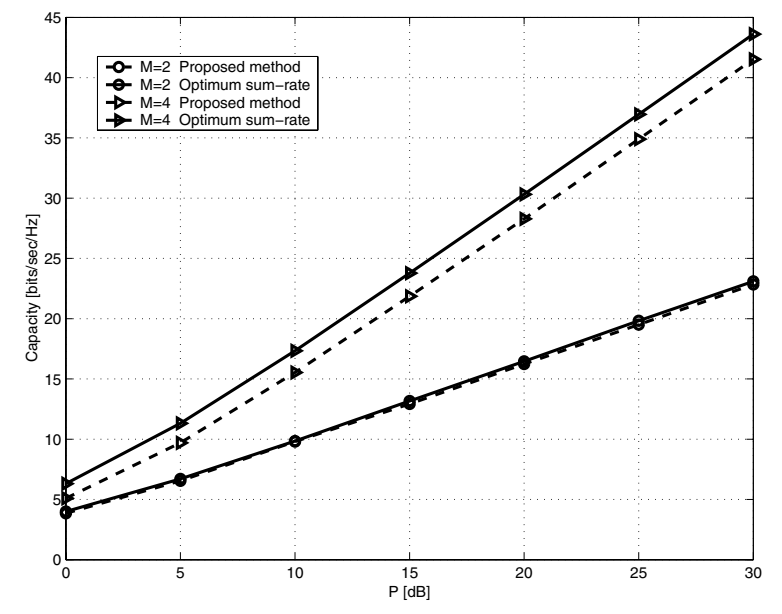

Fig. 5. Sum-rate capacity versus SNR, $N=100, K=1$

value $2 M$ real values must be fed back, the total number of real values required at the base station is asymptotically equal to $\frac{2 M[\log N]^{M}}{\Gamma(M) \Gamma(K)}$. To achieve the optimum performance, the transmitter must have perfect knowledge about all users' channels. Therefore, the total number of real values should be sent to the base station, is equal to $2 N M K$. As a result, the amount of feedback is significantly decreased. We can also decrease the amount of feedback further, by using the modified algorithm in [1].

\section{B. Search complexity}

Since at the first step of the algorithm, only a portion of eigenvectors are pre-selected, the size of search space for next steps is decreased from $N K$ to $L$. As can be observed, at the $m$ th step of the algorithm, the base station searches for the dimension with the smallest $\gamma_{k, j}(m-1)$ among $\mathcal{S}_{m-1}$, which requires $L-m+1$ searches. Therefore, the total number of searches for selecting the desired set is equal to $\sum_{m=1}^{M} L-$ $m+1=M L-\frac{M(M-1)}{2}$, which is linear in $L$. Again, we can restrict our search space if the modified algorithm stated in [1] is used.

As mentioned earlier, the best $M$ eigenvectors for maximizing the sum-rate capacity can be found by exhaustive search. In this case, the size of the search space is equal to $\left(\begin{array}{c}N K \\ M\end{array}\right)$.

In the asymptotic case of $N \rightarrow \infty$, the total number of searches is $\Theta\left([\log N]^{M}\right)$ for the proposed algorithm, which is much less than that of exhaustive search $\left(\Theta\left(N^{M}\right)\right)$. Therefore, using our algorithm the complexity of search at the base station is decreased significantly.

\section{CONCLUSION}

In this paper, we have considered a downlink communication system, in which a base station equipped with $M$ transmit antennas communicates with $N$ users, each equipped with $K$ receive antennas. We have proposed an efficient suboptimum algorithm for selecting a set of users in order to maximize the sum-rate throughput of the system. For the asymptotic case of $N \rightarrow \infty$, it is shown that by using a very simple precoding scheme of zero-forcing beam-forming, the optimum sum-rate which behaves like $M \log \log N$ can be achieved. We have also investigated the complexity of our scheme in terms of the required amount of feedback from the users to the base station, as well as the number of searches needed for selecting the coordinates. It is shown that our algorithm, despite having a very good performance, has a very low complexity.

\section{REFERENCES}

[1] Alireza Bayesteh and Amir K. Khandani, "On the user selection in mimo broadcast channels," Technical Report, University of Waterloo, also available at www.cst.uwaterloo.ca.

[2] E. Telatar, "Capacity of multi-antenna gaussian channels," European Trans. on Telecommunications, vol. 10, no. 6, pp. 585- 595, November 1999.

[3] S. Vishwanath, N. Jindal, and A. Goldsmith, "Duality, achievable rates, and sum-rate capacity of gaussian mimo broadcast channels," IEEE Trans. on Inform. Theory, vol. 49, no. 10, pp. 2658-2668, October 2003.

[4] P. Viswanath and D. N. C. Tse, "Sum capacity of the vector gaussian broadcast channel and uplink-downlink duality," IEEE Trans. on Inform. Theory, vol. 49, pp. 1912-1921, Aug. 2003.

[5] Wei Yu and John Cioffi, "Sum capacity of vector gaussian broadcast channels," accepted in IEEE Transactions on Information Theory.

[6] H. Weingarten, Y. Steinberg, and S. Shamai, "The capacity region of the gaussian mimo broadcast channel," in Conference on Information Sciences and Systems, 2004.

[7] M. Costa, "Writing on dirty paper," IEEE Trans. on Inform. Theory, vol. 29, pp. 439-441, May 1983.

[8] U. Erez, S. Shamai, and R. Zamir, "Capacity and lattice-strategies for cancelling known interference," IEEE Transactions on Information Theory, submitted for Publication., August 2002.

[9] U. Erez and Stephan ten Brink, "Approaching the dirty paper limit for canceling known interference," in 41st Annual Allerton Conference on Communication, Control, and Computing, Oct. 2003.

[10] H. Farmanbar and Amir K. Khandani, "On precoding for channels with known interference at the transmitter," in Proc. Conference on Information Sciences and Systems, The Johns Hopkins University, Baltimore, MD, 2005.

[11] R. Knopp and P. Humblet, "Information capacity and power control in single-cell multiuser communications," in IEEE Int. Conf. Commun., June 1995, vol. 1, pp. 331-335.

[12] P. Viswanath, D. Tse, and R. Laroia, "Opportunistic beamforming using dumb antennas," IEEE Trans. on Inform. theory, vol. 48, pp. 1277-1294, June 2002.

[13] Zhenyu Tu and R. S. Blum, "Multiuser diversity for a dirty paper approach," IEEE Communications Letters, vol. 7, no. 8, pp. 370-372, Aug. 2003.

[14] Mohammad A. Maddah-Ali, Mehdi Ansari, and Amir K. Khandani, "An efficient algorithm for user selection and signalling over mimo multiuser systems," Technical Report UW-ECE 2004-19, 2004.

[15] Derek Gerlach and A. Paulraj, "Adaptive transmitting antenna arrays with feedback," IEEE Signal Processing Letters, vol. 1, no. 10, pp. 150-152, October 1994.

[16] H. Hochwald and S. Vishwanath, "Space-time multiple access: Linear growth in the sum rate," in 40th Annual Allerton Conf. Communications, Control and Computing,, Allerton, IL, Oct. 2002.

[17] G. Caire and S. Shamai, "On the achievable throughput of a multiantenna gaussian broadcast channel," IEEE Trans. on Inform. Theory, vol. 49, pp. 1691 1706,, July 2003.

[18] C. B. Peel, B. M. Hochwald, and A. L. Swindlehurst, "A vectorperturbation technique for near-capacity multiple-antenna multi-user communications-part i: Channel inversion and regularizetion," Submitted to IEEE Trans. on Inform. Theory.

[19] Taesang Yoo and Andrea Goldsmith, "Optimality of zero-forcing beamforming with multiuser diversity," Submitted to ICC 2005.

[20] M. Sharif and B. Hassibi, "On the capacity of mimo broadcast channel with partial side infonnation," in Asilomar Conf. Signals, Systems and Computers,, Nov. 2003, vol. 1, pp. 958-962. 